This is a self-archived version of an original article. This version may differ from the original in pagination and typographic details.

Author(s): Pirinen, Pekka; Suhonen, Jouni; Ydrefors, E.

Title: Charged-current neutrino-nucleus scattering off Xe isotopes

Year: 2019

Version: Published version

Copyright: @ 2019 American Physical Society

Rights: In Copyright

Rights url: http://rightsstatements.org/page//nC/1.0/?language=en

Please cite the original version:

Pirinen, P., Suhonen, J., \& Ydrefors, E. (2019). Charged-current neutrino-nucleus scattering off Xe isotopes. Physical Review C, 99(1), Article 014320.

https://doi.org/10.1103/PhysRevC.99.014320 


\title{
Charged-current neutrino-nucleus scattering off Xe isotopes
}

\author{
P. Pirinen, ${ }^{1}$ J. Suhonen, ${ }^{1}$ and E. Ydrefors ${ }^{2}$ \\ ${ }^{1}$ University of Jyvaskyla, Department of Physics, P. O. Box 35 (YFL), FI-40014, Finland \\ ${ }^{2}$ Instituto Tecnológico de Aeronáutica, DCTA, 12228-900 São José dos Campos, Brazil
}

(Received 14 August 2018; revised manuscript received 14 November 2018; published 25 January 2019)

\begin{abstract}
Xenon detectors are used in the search for dark matter and neutrinoless double-beta decay $(0 v \beta \beta)$. As the next-generation detectors reach masses in the ton scale, neutrinos from astrophysical sources are soon predicted to become background in such detectors. Theoretical predictions of neutrino scattering cross sections and information of nuclear structure effects therein are crucial in accounting for the background. We perform calculations for differential and total cross sections of charged-current neutrino scattering off the most abundant xenon isotopes. The nuclear-structure calculations are made in the proton-neutron quasiparticle random-phase approximation and the microscopic quasiparticle phonon model for even-mass and odd-mass nuclei, respectively. We compute total cross sections as a function of the neutrino energy, and also give estimates of total averaged cross sections for ${ }^{8} \mathrm{~B}$ solar neutrinos and supernova neutrinos using realistic energy distributions.
\end{abstract}

DOI: 10.1103/PhysRevC.99.014320

\section{INTRODUCTION}

Neutrinos provide a fruitful research area for modern physics. The observation of neutrino oscillations has offered a hint of beyond-standard-model physics, and the experimental efforts going into detecting weakly interacting particles are stronger than ever. The opportunities to study the behavior of neutrinos are plentiful, as the Earth is constantly bombarded with neutrinos from astrophysical sources. The Sun, for example, provides a good source of a sizable flux of electron neutrinos. On a rarer occasion, a supernova going off in our galactic neighborhood would result in a huge burst of neutrinos and antineutrinos of all flavors. It has been suggested that observation of the neutrino signal of a supernova burst could be used to solve the neutrino mass hierarchy problem [1,2].

${ }^{136} \mathrm{Xe}$ is known to decay via two-neutrino double-beta decay $(2 \nu \beta \beta)$ [3] and is actively searched for a signal of the elusive neutrinoless double-beta decay $(0 \nu \beta \beta)$ [4]. The EXO-200 detector consists roughly of $80 \%{ }^{136} \mathrm{Xe}$, and close to $20 \%{ }^{134} \mathrm{Xe}[3,4]$. The proposed ton scale successor $\mathrm{nEXO}$ [5], enriched to $90 \%$ in ${ }^{136} \mathrm{Xe}$, presents opportunities also for neutrino physics. Although detecting double-beta decay is its main purpose, it should in addition be able to detect neutrinos of astrophysical origin. Such neutrinos are also expected to be observed as a background in the next-generation dark matter detectors [6,7]. Liquid xenon is used in many dark matter detectors due to its easy scalability, making it possible to expand to larger and larger detector masses. Although the main observed background will be from neutrinos scattering coherently off Xe nuclei, the study of charged-current reactions should also be useful. Our results may therefore be of interest to the XENON [8], LZ [9], and DARWIN [10] collaborations.
Earlier calculations of charged-current neutrino-nucleus scattering cross sections have been carried out for various nuclei in the nuclear shell model [11-16], proton-neutron quasiparticle random-phase approximation (pnQRPA) with realistic effective interactions [17-21], pnQRPA based on Skyrme forces [12,22-24], pnQRPA with neutron-proton pairing [25-27], a consistent relativistic mean-field approach [28], a hybrid model combining shell model for the allowed transitions and pnQRPA for forbidden transitions [29-31], and the microscopic quasiparticle-phonon model (MQPM) [21,32]. In the present work we compute cross sections of neutrinos and antineutrinos scattering off ${ }^{128} \mathrm{Xe},{ }^{129} \mathrm{Xe},{ }^{130} \mathrm{Xe},{ }^{131} \mathrm{Xe},{ }^{132} \mathrm{Xe}$, ${ }^{134} \mathrm{Xe}$, and ${ }^{136} \mathrm{Xe}$ via charged-current processes in the microscopic nuclear frameworks of pnQRPA for the even-mass nuclei and MQPM for the odd-mass nuclei. The calculations are performed using realistic effective interactions.

This paper is an extension of Ref. [20], where the neutralcurrent and charged-current scattering off ${ }^{136} \mathrm{Xe}$ was studied, and of Ref. [33] where we computed cross sections for neutral-current scattering off the same Xe targets as in the present work. Charged-current scattering of neutrinos off ${ }^{132} \mathrm{Xe}$ was also previously calculated in a similar formalism in Ref. [19]. Similar calculations for Cd and Mo nuclei have been carried out in Refs. [21] and [17], respectively. In this work we perform calculations of cross sections as a function of the incoming neutrino energy, and also give estimates of total averaged cross sections for energy distributions representing supernova neutrinos as well as neutrinos from solar ${ }^{8} \mathrm{~B}$ decay.

This paper is organized as follows. In Sec. II we outline the formalism used to compute charged-current neutrino-nucleus scattering. In Sec. III we discuss the essentials of the pnQRPA and MQPM calculations for the involved nuclei. In Sec. IV we discuss details of the obtained cross sections and in Sec. V conclusions are drawn. 


\section{CHARGED-CURRENT NEUTRINO-NUCLEUS SCATTERING}

In this section we outline the ingredients entering the charged-current neutrino-nucleus scattering cross section. Our focus is on processes of the form

$$
\begin{aligned}
& \nu_{l}+(A, Z) \rightarrow l^{-}+(A, Z+1), \\
& \bar{\nu}_{l}+(A, Z) \rightarrow l^{+}+(A, Z-1),
\end{aligned}
$$

where the lepton flavor is labeled by $l=\mathrm{e}, \mu, \tau$. We label the four-momenta of the incoming and outgoing leptons as $k_{\mu}$ and $k_{\mu}^{\prime}$. The momentum transfer to the nucleus is $q_{\mu}=k_{\mu}^{\prime}-k_{\mu}=$ $p_{\mu}-p_{\mu}^{\prime}$, where $p_{\mu}$ and $p_{\mu}^{\prime}$ are the momenta of the nucleus in its initial and final state. The energies of the incoming and outgoing lepton are $E_{k}$ and $E_{k^{\prime}}$

The charged-current neutrino-nucleus scattering differential cross section to a final state of energy $E_{\text {exc }}$ with respect to the initial nuclear state can be written as $[17,34]$

$$
\begin{aligned}
\frac{d^{2} \sigma}{d \Omega d E_{\mathrm{exc}}}= & \frac{G_{\mathrm{F}}^{2} \cos ^{2} \theta_{\mathrm{C}}\left|\mathbf{k}^{\prime}\right| E_{k^{\prime}}}{\pi\left(2 J_{i}+1\right)} F\left( \pm Z_{f}, E_{\mathbf{k}^{\prime}}\right) \\
& \times\left(\sum_{J \geqslant 0} \sigma_{\mathrm{CL}}^{J}+\sum_{J \geqslant 1} \sigma_{\mathrm{T}}^{J}\right),
\end{aligned}
$$

where $F\left( \pm Z, E_{\mathbf{k}^{\prime}}\right)$ is a Fermi function and $\theta_{\mathrm{C}}$ the Cabibbo angle. Adopting the modified effective-momentum approximation of Ref. [35] we define an effective momentum as

$$
k_{\mathrm{eff}}=\sqrt{E_{\mathrm{eff}}^{2}-m_{l}^{2}}
$$

where $m_{l}$ is the mass of the outgoing lepton, and its effective energy is

$$
E_{\text {eff }}=E_{\mathbf{k}^{\prime}}-V_{\mathrm{C}}(0) .
$$

Here $V_{\mathrm{C}}(0)$ is the Coulomb potential produced by the finalstate nucleus at the origin. The Fermi function in Eq. (3) is used only for small effective momenta $k_{\text {eff }} R \ll 1$, where $R$ is the nuclear radius. For higher effective momenta we set $F\left( \pm Z_{f}, E_{\mathbf{k}^{\prime}}\right)=1$ in Eq. (3), and replace the absolute values of the three-momentum and energy of the outgoing lepton by the effective values of Eqs. (4) and (5).

Expression (3) is comprised of the Coulomb-longitudinal $\left(\sigma_{\mathrm{CL}}^{J}\right)$ and transverse $\left(\sigma_{\mathrm{T}}^{J}\right)$ parts. For charged-current reactions they are defined as

$$
\begin{aligned}
\sigma_{\mathrm{CL}}^{J}= & (1+a \cos \theta)\left|\left\langle J_{f}\left\|\mathcal{M}_{J}(q)\right\| J_{i}\right\rangle\right|^{2} \\
& +\left(1+a \cos \theta-2 b \sin ^{2} \theta\right)\left|\left\langle J_{f}\left\|\mathcal{L}_{J}(q)\right\| J_{i}\right\rangle\right|^{2} \\
& +\frac{E_{\mathbf{k}}-E_{\mathbf{k}^{\prime}}}{q}(1+a \cos \theta+c) \\
& \times 2 \operatorname{Re}\left\{\left\langle J_{f}\left\|\mathcal{M}_{J}(q)\right\| J_{i}\right\rangle^{*}\left\langle J_{f}\left\|\mathcal{L}_{J}(q)\right\| J_{i}\right\rangle\right\},
\end{aligned}
$$

and

$$
\begin{aligned}
\sigma_{\mathrm{T}}^{J}= & \left(1-a \cos \theta+b \sin ^{2} \theta\right) \\
& \times\left[\left|\left\langle J_{f} \| \mathcal{T}_{J}^{\mathrm{mag}}(q)|| J_{i}\right\rangle\right|^{2}+\left|\left\langle J_{f}\left\|\mathcal{T}_{J}^{\mathrm{el}}(q)\right\| J_{i}\right\rangle\right|^{2}\right] \\
& \mp \frac{E_{\mathbf{k}}+E_{\mathbf{k}^{\prime}}}{q}(1-a \cos \theta-c) \\
& \times 2 \operatorname{Re}\left\{\left\langle J_{f}|| \mathcal{T}_{J}^{\mathrm{mag}}(q)|| J_{i}\right\rangle\left\langle J_{f}|| \mathcal{T}_{J}^{\mathrm{el}}(q)|| J_{i}\right\rangle^{*}\right\},
\end{aligned}
$$

where the minus sign is taken for neutrino scattering and the plus sign for antineutrino scattering. $J_{i}$ and $J_{f}$ are the initial- and final-state angular momenta of the nucleus and $q$ is the magnitude of the three-momentum transfer. We use the abbreviations

$$
\begin{aligned}
& a=\sqrt{1-\left(m_{l} / E_{\mathbf{k}^{\prime}}\right)^{2}}, \\
& b=\frac{a^{2} E_{\mathbf{k}} E_{\mathbf{k}^{\prime}}}{q^{2}},
\end{aligned}
$$

and

$$
c=\frac{m_{l}^{2}}{q E_{\mathbf{k}^{\prime}}} .
$$

The formalism and various different operators involved are discussed in detail in Refs. [17,34,36,37].

For the computation of averaged total cross sections, we take the supernova neutrino spectrum to be of a two-parameter Fermi-Dirac character

$$
f_{\mathrm{FD}}\left(E_{\mathbf{k}}\right)=\frac{1}{F_{2}\left(\alpha_{v}\right) T_{v}} \frac{\left(E_{\mathbf{k}} / T_{\nu}\right)^{2}}{1+e^{E_{\mathbf{k}} / T_{v}-\alpha_{v}}},
$$

where $\alpha_{v}$ is the so-called pinching parameter, and $T_{v}$ the neutrino temperature. The normalization factor $F_{2}\left(\alpha_{v}\right)$ is defined by the formula

$$
F_{k}\left(\alpha_{v}\right)=\int \frac{x^{k} d x}{1+e^{x-\alpha_{v}}},
$$

and the temperature and mean energy of neutrinos are related by

$$
\frac{\left\langle E_{v}\right\rangle}{T_{v}}=\frac{F_{3}\left(\alpha_{v}\right)}{F_{2}\left(\alpha_{v}\right)} .
$$

Only electron neutrinos partake in charged-current scattering at typical supernova neutrino energies due to the high rest mass of the muon and $\tau$ leptons. However, the supernova environment is suitable for neutrino flavor conversions [38]. The energy distribution of electron neutrinos hitting the earthbound detector can be affected by this neutrino mixing. It should be noted that the behavior of neutrinos inside dense stellar matter is not yet very well understood. In some earlier works the neutrino mixing has been modeled based on the assumption that muon and $\tau$ neutrinos have similar characteristics in supernovae, allowing the problem to be reduced to an effective two-flavor mixing problem $[39,40]$. One such model has been presented and used in earlier similar calculations in Refs. [15,20,21]. Modeling neutrino mixing in this manner leads to a difference in antineutrino-nucleus scattering cross sections between normal and inverted neutrino mass hierarchies, and this result could potentially be used to solve the neutrino mass hierarchy problem. For the xenon nuclei in this work, following the model presented in Refs. [15,20,21] we find that the total cross sections for antineutrino-nucleus scattering are roughly a factor of two higher for normal hierarchy than for inverted hierarchy. However, this is a very simplified model, and the matter should be investigated further elsewhere using neutrino fluxes from most recent supernova simulations and flavor conversion studies. We will focus solely on nonoscillating neutrinos in this work. 
Solar neutrinos from ${ }^{8} \mathrm{~B}$ decay are also of interest for this work. This reaction produces electron neutrinos, which reach the Earth with a flux of roughly $5 \times 10^{6} \mathrm{~cm}^{2} \mathrm{~s}^{-1}$ [41] and large enough energies to be involved in charged-current reactions. The solar neutrino spectrum is better known than the supernova neutrino spectrum. In our calculations we use the ${ }^{8} \mathrm{~B}$ neutrino energy profile from Ref. [42].

\section{NUCLEAR STRUCTURE OF THE TARGET NUCLEI}

In this section we briefly describe the formalism used to compute the nuclear structure of the involved nuclei. We have performed computations in the proton-neutron quasiparticle random-phase approximation (pnQRPA), and microscopic quasiparticle-phonon model (MQPM). The charge conserving QRPA results needed for the MQPM calculations were described in Ref. [33], where we computed neutral-current neutrino-nucleus scattering off the same Xe isotopes as in the present work.

\section{A. pnQRPA calculations}

We computed the nuclear structure of the odd-odd isotopes in the charge-changing pnQRPA framework. In the calculations an even-even Xe nucleus is taken as a reference nucleus, from which the states of the neighboring odd-odd Cs and I nuclei are obtained as simple phonon excitations. The basic excitations with respect to the pnQRPA vacuum are created with the phonon creation operator

$$
Q_{\omega}^{\dagger}=\sum_{p n}\left(X_{p n}^{\omega}\left[a_{p}^{\dagger} a_{n}^{\dagger}\right]_{J_{\omega} M_{\omega}}+Y_{p n}^{\omega}\left[\tilde{a}_{p} \tilde{a}_{n}\right]_{J_{\omega} M_{\omega}}\right)
$$

for an excited state $\omega=\left(J_{\omega}, M_{\omega}, \pi_{\omega}, k_{\omega}\right)$, where $k_{\omega}$ is a number labeling the excited states of given $J^{\pi} . X_{a b}^{\omega}$, and $Y_{a b}^{\omega}$ are amplitudes obtained by solving the eigenvalue problem of the pnQRPA equations

$$
\left[\begin{array}{cc}
\mathrm{A} & \mathrm{B} \\
-\mathrm{B}^{*} & -\mathrm{A}^{*}
\end{array}\right]\left[\begin{array}{c}
\mathrm{X}^{\omega} \\
\mathrm{Y}^{\omega}
\end{array}\right]=E_{\omega}\left[\begin{array}{c}
\mathrm{X}^{\omega} \\
\mathrm{Y}^{\omega}
\end{array}\right]
$$

where the matrices A and B are defined in detail in Ref. [43].

We perform the pnQRPA calculations using the same large model space as in Ref. [33]. The valence space consists of the entire $0 s-0 d, 1 p-0 f-0 g, 2 s-1 d-0 h$, and $1 f-2 p$ major shells, and we also add the $0 i_{13 / 2}$ and $0 i_{11 / 2}$ orbitals. The single-particle bases are constructed by solving the Schrödinger equation for a Coulomb-corrected Woods-Saxon potential. The Bonn A one-boson exchange potential [46] was used to estimate the residual two-body interaction. The Woods-Saxon parameters used are those of Ref. [44], which have been fitted to work quite well for nuclei close to the line of stability. Using this parametrization, the computed quasiparticle energies with respect to the BCS vacuum for the orbitals near the Fermi level are also in reasonable agreement with experimental energy levels of the odd-mass neighbors of the reference nucleus. However, the agreement gets slightly worse for xenon isotopes with increasing mass number, and for ${ }^{136} \mathrm{Xe}$ we were forced to adjust the single-particle levels to maintain reliability in our calculations. We adopt the set of adjusted single-particle energies from Ref. [20]. For these the RMS deviation from the Woods-Saxon single-particle energies is $1.04 \mathrm{MeV}$. Single-particle energies have also been produced by using self-consistent mean-field approaches, like the one of Ref. [45] using several different parametrizations of the Skyrme interactions. In Ref. [45] single-particle energies were computed near the Fermi surface of nuclei of various masses and the results were compared with data. The mean deviation from the experimental values was found to be $2.11-3.66 \mathrm{MeV}$, which is much more than the necessary adjustments for the $A=136$ case in the present work. Overall, with our presently used single-particle energies we reproduce reasonably well the low-energy spectra of the neutron-odd and proton-odd nuclei next to the even Xe isotopes discussed in this work.

Several model parameters in the quasiparticle framework need to be fixed by fitting computed observables to experimental values. In the BCS calculation we fit the proton and neutron pairing strengths $A_{\text {pair }}^{\mathrm{p}}$ and $A_{\text {pair }}^{\mathrm{n}}$ so that the lowest quasiparticle energy matches the empirical pairing gap given by the three-point formula [47]:

$$
\begin{aligned}
\Delta_{\mathrm{p}}(A, Z)= & \frac{1}{4}(-1)^{Z+1}\left[S_{\mathrm{p}}(A+1, Z+1)\right. \\
& \left.-2 S_{\mathrm{p}}(A, Z)+S_{\mathrm{p}}(A-1, Z-1)\right], \\
\Delta_{\mathrm{n}}(A, Z)= & \frac{1}{4}(-1)^{A-Z+1}\left[S_{\mathrm{n}}(A+1, Z)\right. \\
& \left.-2 S_{\mathrm{n}}(A, Z)+S_{\mathrm{n}}(A-1, Z)\right] .
\end{aligned}
$$

It should be noted that for the neutron-magic ${ }^{136} \mathrm{Xe}$ this procedure cannot be applied to the neutron pairing strength. We have instead used a bare value of $A_{\text {pair }}^{\mathrm{n}}=1.0$ for ${ }^{136} \mathrm{Xe}$, i.e., the orbitals below the neutron Fermi surface have BCS occupation amplitudes $u=0, v=1$, and those above the Fermi surface have $u=1, v=0$.

Neutrino-nucleus scattering calculations suffer from the ambiguity of the weak axial-vector coupling constant $g_{\mathrm{A}}$. While the free-nucleon value of $g_{\mathrm{A}}$ is approximately 1.27 , sizable quenching of $g_{\mathrm{A}}$ has been observed in pnQRPA calculations of single- and double-beta decays, see Ref. [48] for a review. In the mass range of $A=100-134$ an average value as low as $g_{\mathrm{A}} \approx 0.60$ was observed in Ref. [49] by studying single-beta decays. The studies of quenching of $g_{\mathrm{A}}$ usually examine ground-state-to-ground-state transitions. However, the transitions in neutrino-nucleus scattering often involve highlying states and the quenching of $g_{\mathrm{A}}$ could well be different in the transitions to these states. The quenching of $g_{\mathrm{A}}$ can also be different for different multipole transitions [48]. Thus we use the value $g_{\mathrm{A}}=1.00$ as a conservative estimate, but we also show results for $g_{\mathrm{A}}=0.7$ to give a realistic interval of the effect of this quenching in total neutrino-nucleus scattering

TABLE I. The values of the pinching parameter $\alpha$, average neutrino energy $\left\langle E_{\mathbf{k}}\right\rangle$, and neutrino temperature entering the Fermi-Dirac distribution. Parameter values are given for electron neutrinos and antineutrinos $v_{e}, \bar{v}_{e}$.

\begin{tabular}{lccc}
\hline \hline Flavor & $\alpha_{v}$ & $\left\langle E_{\mathbf{k}}\right\rangle(\mathrm{MeV})$ & $T_{v}(\mathrm{MeV})$ \\
\hline$\nu_{e}$ & 3.0 & 11.5 & 2.88 \\
$\bar{v}_{e}$ & 3.0 & 13.6 & 3.41 \\
\hline \hline
\end{tabular}


TABLE II. Total charged-current scattering cross section for electron neutrinos scattering off xenon targets as a function of neutrino energy. The cross sections are given in units of $\mathrm{cm}^{2}$. We show results with $g_{\mathrm{A}}=1.0$, and also for $g_{\mathrm{A}}=0.7$ in parentheses.

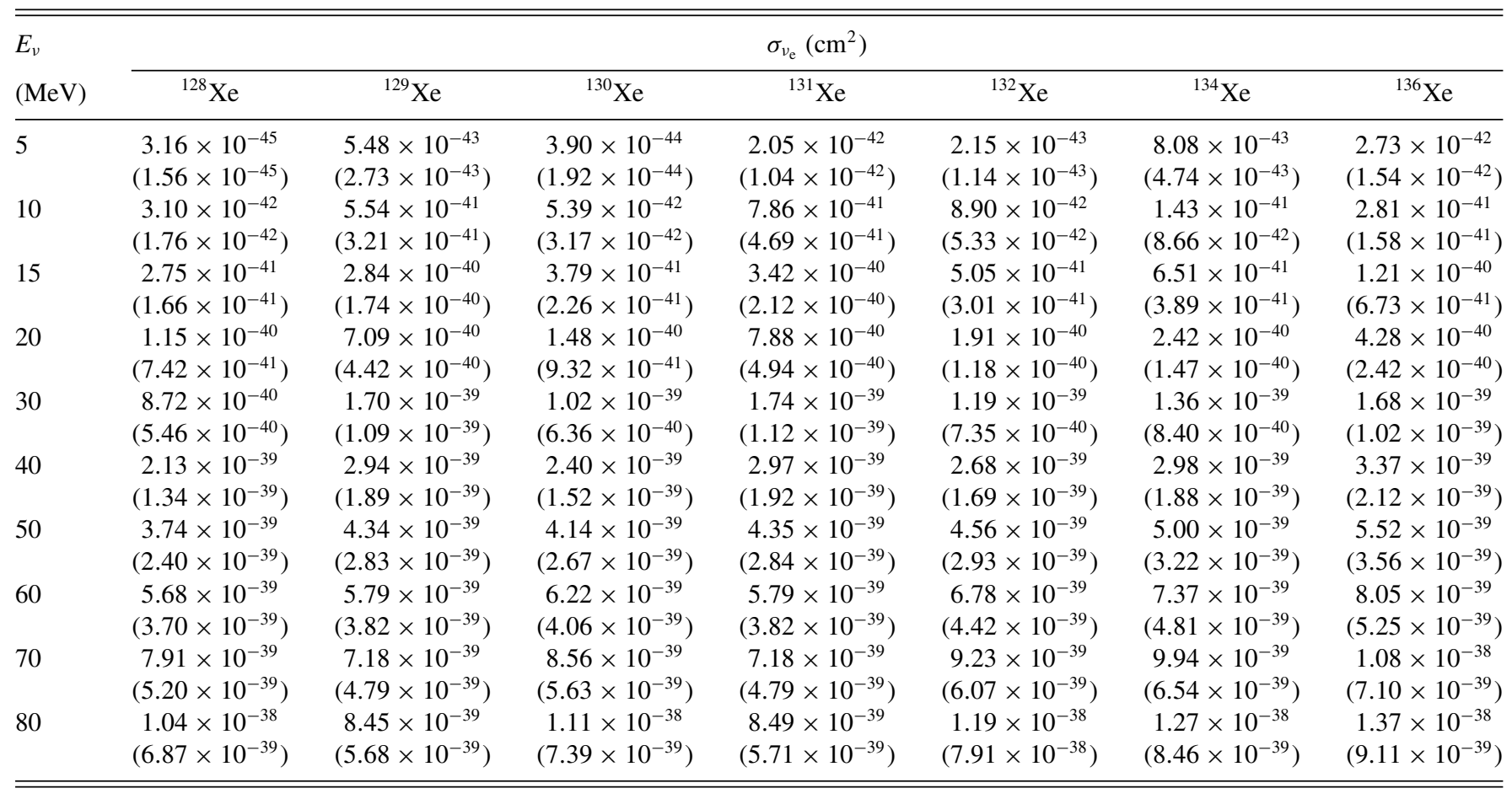

cross sections. This value is justified by the single-beta-decay studies of Refs. [49,50] that show $g_{\mathrm{A}} \approx 0.7$ to be a realistic choice for nuclei in the mass range $A=128-134$.

The particle-particle and particle-hole parts of the matrices $\mathrm{A}$ and $\mathrm{B}$ in Eq. (15) for the $1^{+}$states are scaled by strength parameters $g_{\mathrm{pp}}$ and $g_{\mathrm{ph}}$, respectively. The $\log f t$ value of the Gamow-Teller $\beta$ decay from the first $1^{+}$state is heavily dependent on $g_{\text {pp }}$, while the energy of the Gamow-Teller giant resonance (GTGR) is determined by the value of $g_{\mathrm{ph}}$ [43]. To fix the value of $g_{\mathrm{ph}}$ we fit the energy of the centroid

TABLE III. Same as Table II but for antineutrinos.

\begin{tabular}{|c|c|c|c|c|c|c|c|}
\hline $\begin{array}{l}E_{\bar{v}} \\
(\mathrm{MeV})\end{array}$ & \multicolumn{7}{|c|}{$\sigma_{\bar{\nu}_{\mathrm{e}}}\left(\mathrm{cm}^{2}\right)$} \\
\hline 5 & $\begin{array}{c}1.38 \times 10^{-48} \\
\left(6.61 \times 10^{-49}\right)\end{array}$ & $\begin{array}{c}2.47 \times 10^{-45} \\
\left(1.30 \times 10^{-45}\right)\end{array}$ & $\begin{array}{c}4.40 \times 10^{-50} \\
\left(2.09 \times 10^{-50}\right)\end{array}$ & $\begin{array}{c}1.76 \times 10^{-44} \\
\left(8.60 \times 10^{-45}\right)\end{array}$ & $\begin{array}{c}9.12 \times 10^{-51} \\
\left(4.53 \times 10^{-51}\right)\end{array}$ & $\begin{array}{c}0.0 \\
(0.0)\end{array}$ & $\begin{array}{c}0.0 \\
(0.0)\end{array}$ \\
\hline 10 & $\begin{array}{c}1.60 \times 10^{-44} \\
\left(1.03 \times 10^{-44}\right)\end{array}$ & $\begin{array}{c}1.78 \times 10^{-43} \\
\left(9.85 \times 10^{-44}\right)\end{array}$ & $\begin{array}{c}8.55 \times 10^{-45} \\
\left(5.75 \times 10^{-45}\right)\end{array}$ & $\begin{array}{c}1.63 \times 10^{-43} \\
\left(8.31 \times 10^{-44}\right)\end{array}$ & $\begin{array}{c}6.38 \times 10^{-45} \\
\left(4.39 \times 10^{-45}\right)\end{array}$ & $\begin{array}{c}4.88 \times 10^{-45} \\
\left(3.41 \times 10^{-45}\right)\end{array}$ & $\begin{array}{c}9.51 \times 10^{-46} \\
\left(5.84 \times 10^{-46}\right)\end{array}$ \\
\hline 20 & $\begin{array}{c}1.30 \times 10^{-42} \\
\left(8.22 \times 10^{-43}\right)\end{array}$ & $\begin{array}{c}3.74 \times 10^{-42} \\
\left(2.13 \times 10^{-42}\right)\end{array}$ & $\begin{array}{c}1.03 \times 10^{-42} \\
\left(6.53 \times 10^{-43}\right)\end{array}$ & $\begin{array}{c}2.70 \times 10^{-42} \\
\left(1.47 \times 10^{-42}\right)\end{array}$ & $\begin{array}{c}8.38 \times 10^{-43} \\
\left(5.30 \times 10^{-43}\right)\end{array}$ & $\begin{array}{c}6.44 \times 10^{-43} \\
\left(4.07 \times 10^{-43}\right)\end{array}$ & $\begin{array}{c}3.66 \times 10^{-43} \\
\left(2.25 \times 10^{-43}\right)\end{array}$ \\
\hline 30 & $\begin{array}{c}7.81 \times 10^{-42} \\
\left(4.73 \times 10^{-42}\right)\end{array}$ & $\begin{array}{c}1.69 \times 10^{-41} \\
\left(9.51 \times 10^{-42}\right)\end{array}$ & $\begin{array}{c}6.62 \times 10^{-42} \\
\left(3.98 \times 10^{-42}\right)\end{array}$ & $\begin{array}{c}1.29 \times 10^{-41} \\
\left(7.05 \times 10^{-42}\right)\end{array}$ & $\begin{array}{c}5.68 \times 10^{-42} \\
\left(3.39 \times 10^{-42}\right)\end{array}$ & $\begin{array}{c}4.75 \times 10^{-42} \\
\left(2.79 \times 10^{-42}\right)\end{array}$ & $\begin{array}{c}3.53 \times 10^{-42} \\
\left(2.00 \times 10^{-42}\right)\end{array}$ \\
\hline 40 & $\begin{array}{c}2.40 \times 10^{-41} \\
\left(1.43 \times 10^{-41}\right)\end{array}$ & $\begin{array}{c}4.50 \times 10^{-41} \\
\left(2.52 \times 10^{-41}\right)\end{array}$ & $\begin{array}{c}2.11 \times 10^{-41} \\
\left(1.24 \times 10^{-41}\right)\end{array}$ & $\begin{array}{c}3.58 \times 10^{-41} \\
\left(1.96 \times 10^{-41}\right)\end{array}$ & $\begin{array}{c}1.87 \times 10^{-41} \\
\left(1.09 \times 10^{-41}\right)\end{array}$ & $\begin{array}{c}1.63 \times 10^{-41} \\
\left(9.35 \times 10^{-42}\right)\end{array}$ & $\begin{array}{c}1.35 \times 10^{-41} \\
\left(7.57 \times 10^{-42}\right)\end{array}$ \\
\hline 70 & $\begin{array}{c}2.34 \times 10^{-40} \\
\left(1.37 \times 10^{-40}\right)\end{array}$ & $\begin{array}{c}3.53 \times 10^{-40} \\
\left(1.99 \times 10^{-40}\right)\end{array}$ & $\begin{array}{c}2.14 \times 10^{-40} \\
\left(1.24 \times 10^{-40}\right)\end{array}$ & $\begin{array}{c}2.94 \times 10^{-40} \\
\left(1.63 \times 10^{-40}\right)\end{array}$ & $\begin{array}{c}1.97 \times 10^{-40} \\
\left(1.13 \times 10^{-40}\right)\end{array}$ & $\begin{array}{c}1.81 \times 10^{-40} \\
\left(1.02 \times 10^{-40}\right)\end{array}$ & $\begin{array}{c}1.68 \times 10^{-40} \\
\left(9.30 \times 10^{-41}\right)\end{array}$ \\
\hline 80 & $\begin{array}{c}4.02 \times 10^{-40} \\
\left(2.36 \times 10^{-40}\right)\end{array}$ & $\begin{array}{c}5.55 \times 10^{-40} \\
\left(3.17 \times 10^{-40}\right)\end{array}$ & $\begin{array}{c}3.72 \times 10^{-40} \\
\left(2.16 \times 10^{-40}\right)\end{array}$ & $\begin{array}{c}4.69 \times 10^{-40} \\
\left(2.62 \times 10^{-40}\right)\end{array}$ & $\begin{array}{c}3.47 \times 10^{-40} \\
\left(1.99 \times 10^{-40}\right)\end{array}$ & $\begin{array}{c}3.22 \times 10^{-40} \\
\left(1.82 \times 10^{-40}\right)\end{array}$ & $\begin{array}{c}3.03 \times 10^{-40} \\
\left(1.69 \times 10^{-40}\right)\end{array}$ \\
\hline
\end{tabular}



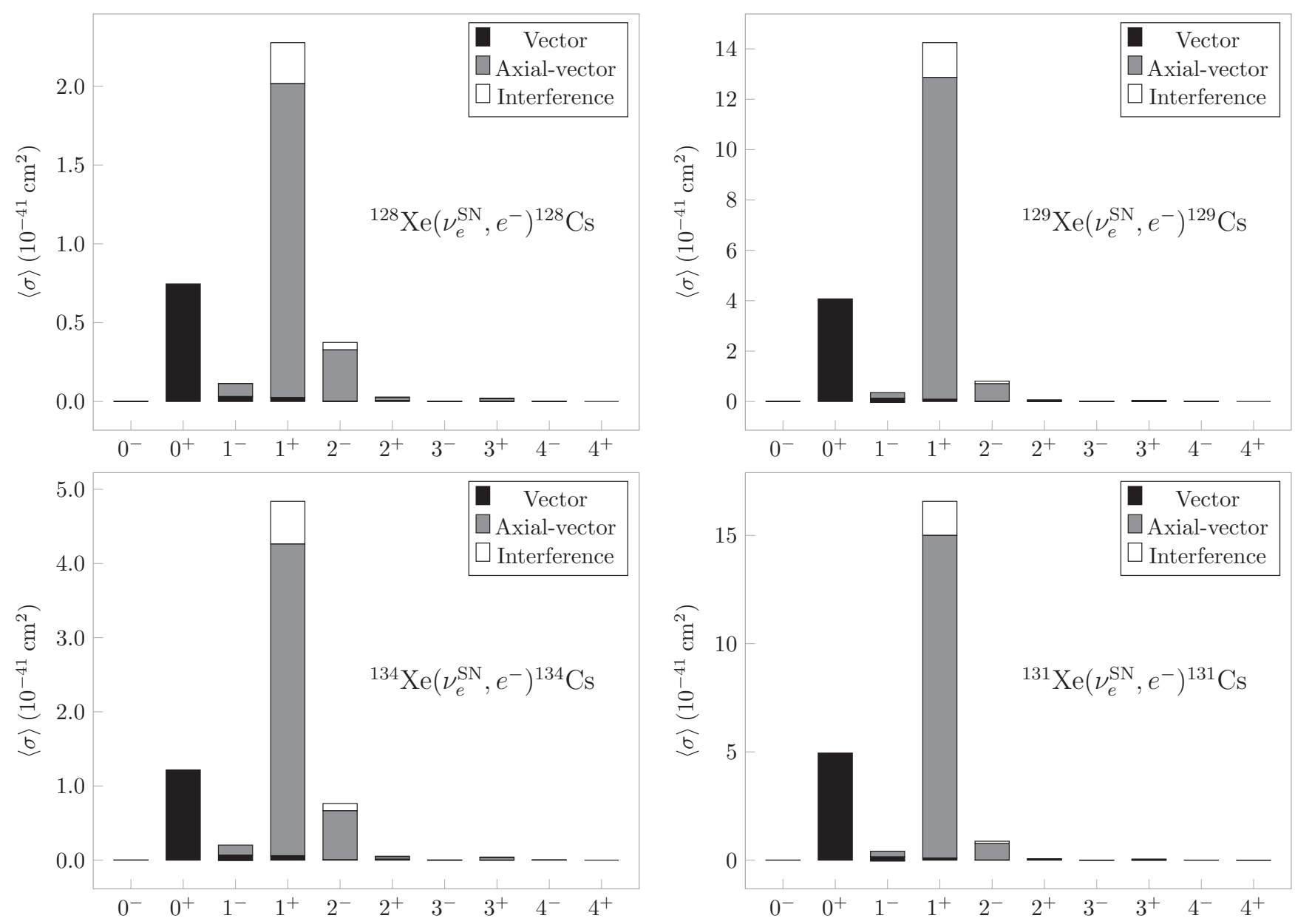

FIG. 1. Contributions of different multipole channels to the total folded cross section for supernova electron neutrinos scattering off a representative sample of Xe targets. Cross sections are given in units of $10^{-41} \mathrm{~cm}^{2}$ in all panels. Results are shown for ${ }^{128} \mathrm{Xe}\left(\right.$ top left), ${ }^{129} \mathrm{Xe}$ (top right), ${ }^{134} \mathrm{Xe}$ (bottom left), and ${ }^{131} \mathrm{Xe}$ (bottom right). The calculations were made using $g_{\mathrm{A}}=1.0$.

of the GTGR to an empirical value following the procedure described in detail in Ref. [43]. For ${ }^{136} \mathrm{Xe}$ we have used the experimental GTGR location of $E_{\mathrm{GTGR}}\left({ }^{136} \mathrm{Xe}\right) \approx 15 \mathrm{MeV}$ given in Ref. [51].

In the analysis of Refs. [52,53] an average value of $g_{\mathrm{pp}}=0.63 \pm 0.17$ was obtained for a range of medium-heavy

TABLE IV. Total folded cross section in units of $\mathrm{cm}^{2}$ for supernova neutrinos and antineutrinos scattering off xenon targets. The results are shown for neutrinos in columns 2 and 3 for $g_{\mathrm{A}}=1.0$ and $g_{\mathrm{A}}=0.7$, respectively, and similarly for antineutrinos in columns 4 and 5 .

\begin{tabular}{lccccc}
\hline \hline Target & \multicolumn{2}{c}{$\sigma_{v_{\mathrm{e}}}\left(\mathrm{cm}^{2}\right)$} & & \multicolumn{2}{c}{$\sigma_{\overline{\mathrm{v}}_{\mathrm{e}}}\left(\mathrm{cm}^{2}\right)$} \\
\cline { 2 - 3 } \cline { 5 - 6 } & $g_{\mathrm{A}}=1.0$ & $g_{\mathrm{A}}=0.7$ & & $g_{\mathrm{A}}=1.0$ & $g_{\mathrm{A}}=0.7$ \\
\hline${ }^{128} \mathrm{Xe}$ & $3.56 \times 10^{-41}$ & $2.23 \times 10^{-41}$ & & $7.72 \times 10^{-43}$ & $4.77 \times 10^{-43}$ \\
${ }^{129} \mathrm{Xe}$ & $1.95 \times 10^{-40}$ & $1.20 \times 10^{-40}$ & & $2.00 \times 10^{-42}$ & $1.13 \times 10^{-42}$ \\
${ }^{130} \mathrm{Xe}$ & $4.52 \times 10^{-41}$ & $2.79 \times 10^{-41}$ & & $6.31 \times 10^{-43}$ & $3.89 \times 10^{-43}$ \\
${ }^{131} \mathrm{Xe}$ & $2.29 \times 10^{-40}$ & $1.42 \times 10^{-40}$ & & $1.50 \times 10^{-42}$ & $8.16 \times 10^{-43}$ \\
${ }^{132} \mathrm{Xe}$ & $5.71 \times 10^{-41}$ & $3.49 \times 10^{-41}$ & & $5.29 \times 10^{-43}$ & $3.24 \times 10^{-43}$ \\
${ }^{134} \mathrm{Xe}$ & $7.12 \times 10^{-41}$ & $4.33 \times 10^{-41}$ & & $4.29 \times 10^{-43}$ & $2.60 \times 10^{-43}$ \\
${ }^{136} \mathrm{Xe}$ & $1.16 \times 10^{-40}$ & $6.67 \times 10^{-41}$ & & $2.89 \times 10^{-43}$ & $1.69 \times 10^{-43}$ \\
\hline \hline
\end{tabular}

nuclei. In Ref. [49] values of $0.6 \leqslant g_{\text {pp }} \leqslant 0.8$ were deemed suitable for mass $A=100-134$ isotopes by examining $\beta$ decay properties. In this work we choose to use the value of $g_{\mathrm{pp}}=0.60$, which reproduces very well the known $\log f t$ value of the $\beta^{-}$decay of ${ }^{128} \mathrm{I}$ in our pnQRPA calculation. It should be noted, however, that the average cross sections computed in this work are not very sensitive to the value of $g_{\mathrm{pp}}$.

Most of the transition strength in the case of the Fermi operator is carried by a transition from a $0^{+}$ground state of an even-even nucleus to the $0^{+}$isobaric analog state (IAS). In the pnQRPA the IAS is always predicted too low in energy [54]. If left uncorrected, this leads to overestimations in neutrinoscattering cross sections. Therefore we have set the energy of the IAS in the final-state Cs nuclei by hand to match the empirical value given by Ref. [55]. The empirical energy for the IAS was set only for the calculation of cross sections. For ${ }^{136} \mathrm{Cs}$ we use the experimental value of $E_{\mathrm{IAS}}\left({ }^{136} \mathrm{Cs}\right)=$ 13.38 MeV, taken from Ref. [51]. For ${ }^{136} \mathrm{Cs}$ the empirical formula of Ref. [55] gives an energy of $12.89 \mathrm{MeV}$, giving an estimate of its accuracy. The total averaged cross section is not very sensitive to the energy of the IAS if $E_{\mathrm{IAS}} \gtrsim 10 \mathrm{MeV}$. After the corrections this inequality holds for all the examined 

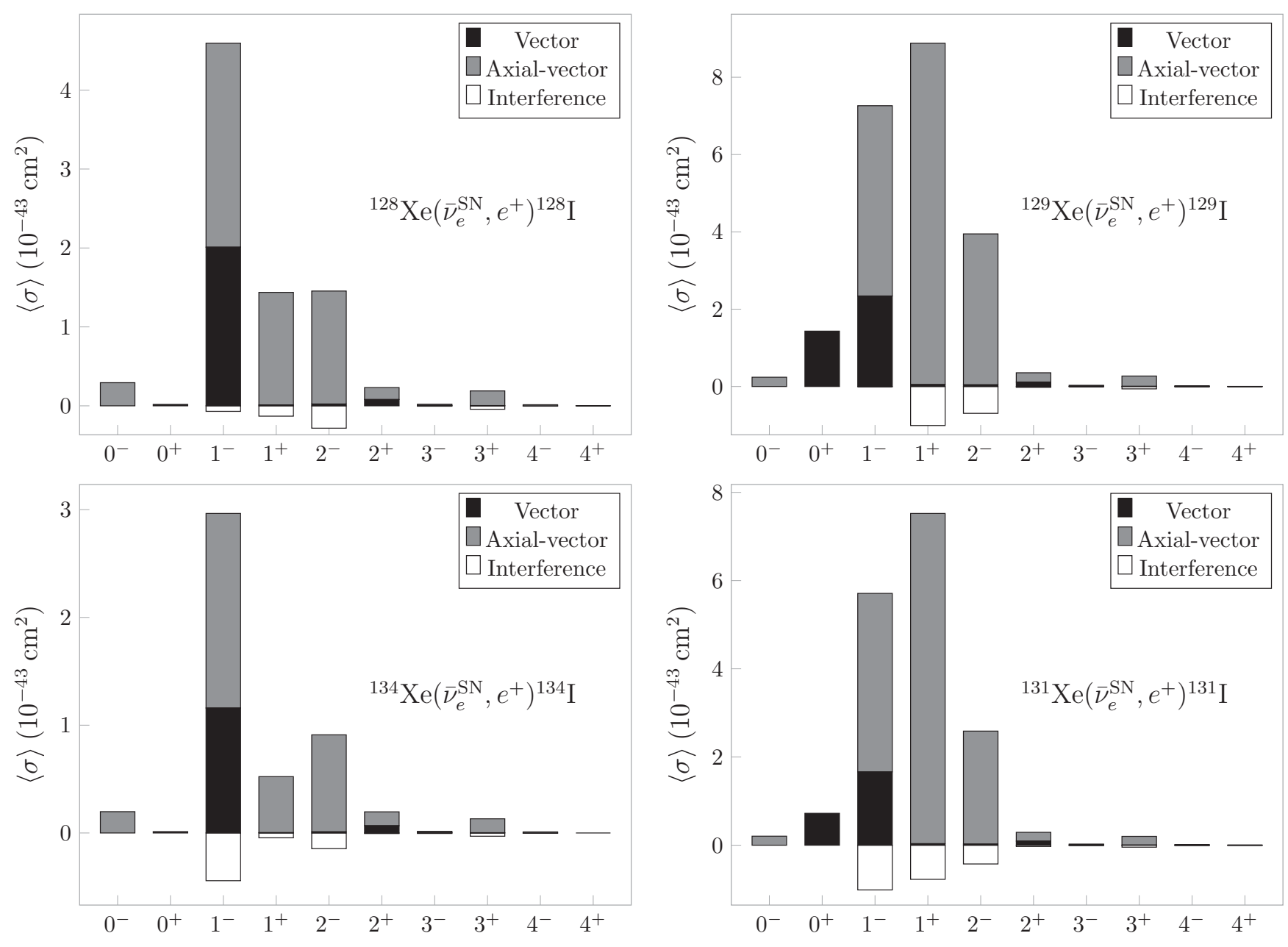

FIG. 2. Same as Fig. 1 but for supernova electron antineutrinos.

cases but ${ }^{128} \mathrm{Xe}$, where the IAS is predicted to be at $E_{\mathrm{IAS}}=$ 9.30 MeV.

\section{B. MQPM calculations}

Nuclear structure calculations for odd-mass nuclei were performed by using the MQPM formalism, in which we account for three-quasiparticle configurations by coupling a single quasiparticle with a two-quasiparticle QRPA phonon. Details of the QRPA calculations used in this work are given in Ref. [33]. The MQPM basic excitation can be written as [56]

$$
\Gamma_{k}^{\dagger}(j m)=\sum_{n} C_{n}^{k} a_{n j m}^{\dagger}+\sum_{a, \omega} D_{a \omega}^{k}\left[a_{a}^{\dagger} Q_{\omega}^{\dagger}\right]_{j m},
$$

where $Q_{\omega}^{\dagger}$ is the phonon creation operator of the charge conserving QRPA. The amplitudes $C$ and $D$ are computed by solving the MQPM equations of motion. The detailed description of the process can be found in Ref. [56]. All QRPA phonons of $J \leqslant 6$ and an energy less than $10 \mathrm{MeV}$ are selected to be used in the MQPM calculation. We base our computations of initial and final nuclear scattering states on the same reference nucleus. For example, in the
${ }^{129} \mathrm{Xe}+v \rightarrow{ }^{129} \mathrm{Cs}+e^{-}$reaction the reference nucleus is ${ }^{128} \mathrm{Xe}$ for both ${ }^{129} \mathrm{Xe}$ and ${ }^{129} \mathrm{Cs}$.

The first excited $0^{+}$state [18,21] and the first $1^{-}$state [43] in the QRPA calculations are known to be spurious. These states have therefore been omitted in the MQPM calculations of this work.

\section{NEUTRINO SCATTERING RESULTS}

We have computed total cross sections for charged-current neutrino-nucleus scattering as a function of the neutrino energy, and also averaged total cross sections for solar ${ }^{8} \mathrm{~B}$ neutrinos and supernova neutrinos scattering off the most abundant xenon isotopes. For the average supernova neutrino energies $\left\langle E_{\mathbf{k}}\right\rangle$ and pinching parameters $\alpha$ we follow the choices of Ref. [20] presented in Table I for electron neutrinos and antineutrinos. These parameters are used in the Fermi-Dirac distribution of supernova (anti)neutrinos throughout the paper.

We will first examine the cross sections as a function of the neutrino energy, independent of any specific energy distribution. The calculated cross sections of the chargedcurrent processes for neutrinos are presented in Table II and for antineutrinos in Table III. We show results for $g_{\mathrm{A}}=1.0$ and $g_{\mathrm{A}}=0.7$, as discussed in Sec. III A. The cross sections 

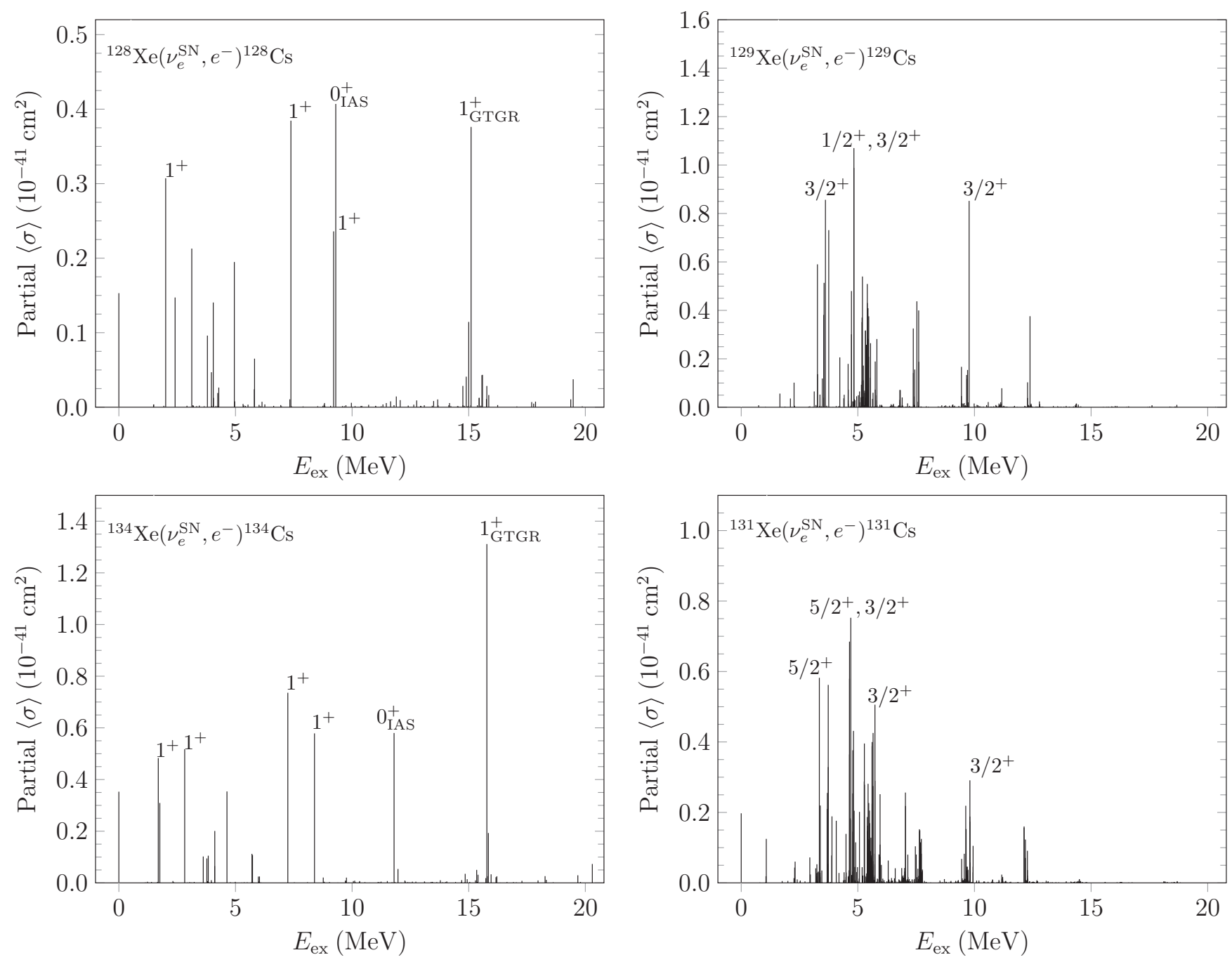

FIG. 3. Main contributions to the total averaged cross section arising from different final states of the reaction daughter Cs nuclei in the reactions ${ }^{A} \mathrm{Xe}+v_{e}^{\mathrm{SN}} \rightarrow{ }^{A} \mathrm{Cs}+e^{-}$. Results are shown for $A=128$ (top left), $A=129$ (top right), $A=134$ (bottom left), and $A=131$ (bottom right). The calculations were made using $g_{\mathrm{A}}=1.0$.

increase rapidly with increasing neutrino energy. We also notice an increasing (decreasing) trend in the cross sections for neutrinos (antineutrinos) with increasing mass number of the Xe isotopes. This is in part a manifestation of the $Q$ value of the neutrino (antineutrino) scattering process decreasing (increasing) when moving towards the heaviest stable xenon isotope ${ }^{136} \mathrm{Xe}$. Moreover, the Gamow-Teller transition strength in the pnQRPA fulfils the Ikeda sum rule, which states that $S_{-}\left(1^{+}\right)-S_{+}\left(1^{+}\right)=3(N-Z)$, where $S_{ \pm}$is the sum of the $\beta^{ \pm}$type, i.e., $(p \rightarrow n)$ or $(n \rightarrow p)$ type transition strength to each possible final state [43]. A similar sum rule holds for the Fermi transitions as $S_{-}\left(0^{+}\right)-S_{+}\left(0^{+}\right)=N-Z$. As the transition strength to the $\beta^{+}$direction is very small for the Xe nuclei, it is expected that the important $1^{+}$and $0^{+}$strengths in the neutrino-scattering reactions of Eq. (1) scale roughly as $N-Z$ for low momentum transfer, which provides another source for the increase in the cross sections as $\mathrm{N}$ increases for the $\mathrm{Xe}$ isotopes. The $1^{+}$and $0^{+}$strengths in antineutrino-scattering are instead relatively larger for the lighter Xe isotopes, which contributes to the decreasing behavior with increasing $N$. These two effects seem to be the dominant drivers of the isotopic dependence of the cross sections.

For the odd-mass isotopes the cross sections are larger than those for even-mass isotopes for neutrino energies under roughly $30 \mathrm{MeV}$ but the situation is reversed for higher energies. This favorable enhancement in the low-neutrino-energy region is likely to be due to a combination of the low $Q$ value of the transition and the denser low-lying energy spectra of the odd-mass nuclei. As the ground-state angular momentum of the odd-mass nuclei is nonzero, there is also more freedom of angular-momentum coupling to these numerous low-lying final states. For antineutrinos the cross sections for odd-mass nuclei are larger than cross sections for even nuclei across the entire energy range. Cross sections of monoenergetic neutrinos and antineutrinos can be read off the tables directly, but for a distribution of neutrino energies a folding procedure must be followed.

We can compare our results for the $A=136$ case with those of Ref. [20], where $g_{\mathrm{A}}=1.0$ was used. The calculations 

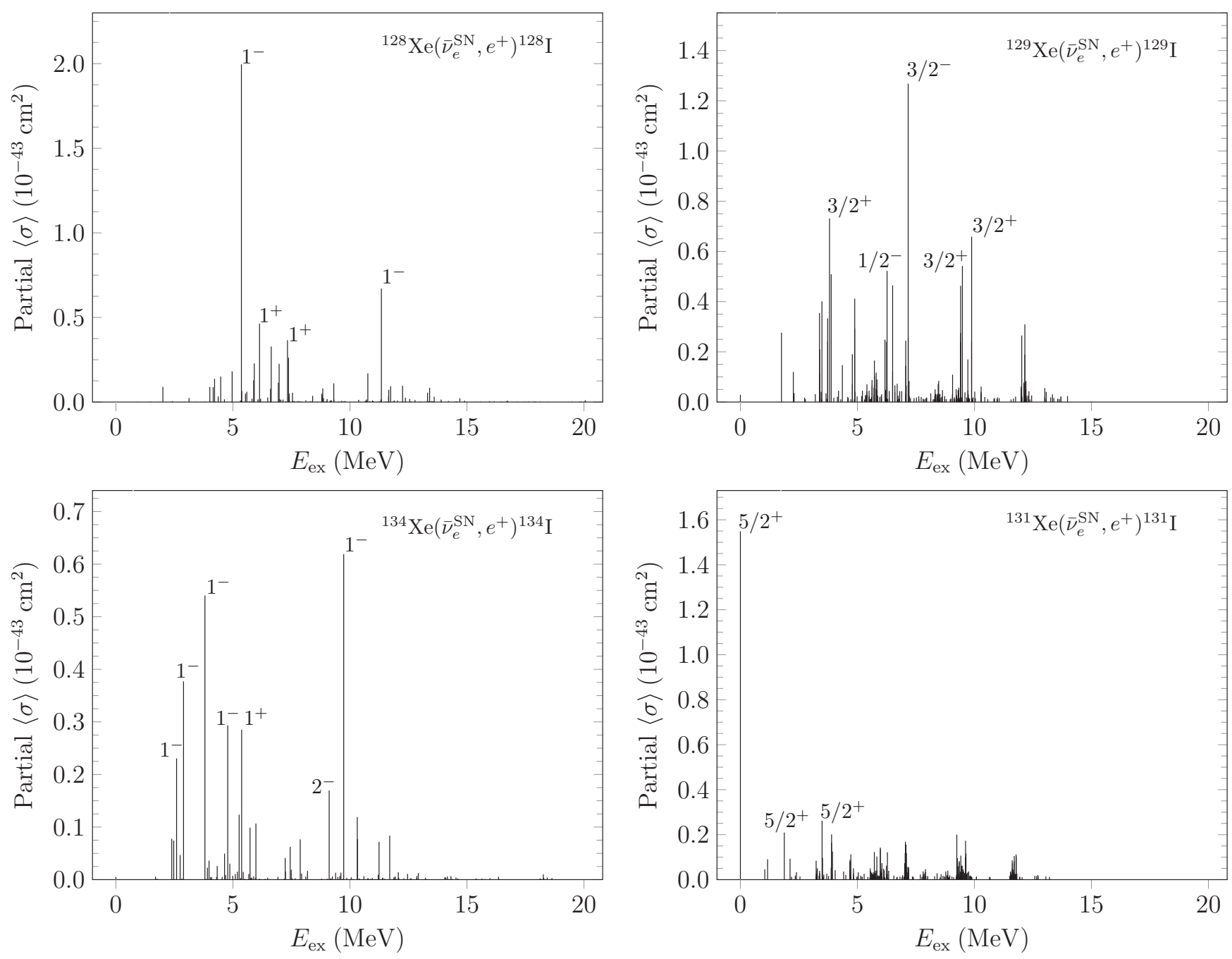

FIG. 4. Main contributions to the total averaged cross section arising from different final states of the reaction daughter I nuclei in the reactions ${ }^{A} \mathrm{Xe}+\bar{v}_{e}^{\mathrm{SN}} \rightarrow{ }^{A} \mathrm{I}+e^{+}$. Results are shown for $A=128$ (top left), $A=129$ (top right), $A=134$ (bottom left), and $A=131$ (bottom right). The calculations were made using $g_{\mathrm{A}}=1.0$.

were essentially the same, but for the antineutrino scattering process the $Q$ value used in the calculations of Ref. [20] was roughly an $\mathrm{MeV}$ lower than the experimental value of $Q=6.930 \mathrm{MeV}$ used in this work. Therefore our cross sections for neutrinos are exactly the same as in Ref. [20], but for antineutrinos our results are smaller especially for low neutrino energies.

Cross sections for the $A=132$ case have been calculated in the pnQRPA formalism in Ref. [19]. The cross sections obtained in Ref. [19] for neutrinos appear to be larger than the present work for neutrino energies below $40 \mathrm{MeV}$. For antineutrinos the cross sections of Ref. [19] are significantly larger than ours for all energies. In the present work the energy of the IAS, which is predicted very low by the pnQRPA, has been corrected to a reasonable empirical value as explained in Sec. III A. In Ref. [19] no mention of such a procedure can be found. Similarly we have fixed the parameter $g_{\mathrm{ph}}$ so that the energy of the GTGR matches its empirical location. In Ref. [19] a bare value of $g_{\mathrm{ph}}=1.00$ has been used for the $1^{+}$states, whereas a value of $g_{\mathrm{ph}}=1.60$ was required in the present work. Therefore the contributions arising from the $0^{+}$ IAS and $1^{+}$GTGR may be overestimated in Ref. [19].

TABLE V. Total averaged cross section $\langle\sigma\rangle$ of charged-current scattering of solar ${ }^{8} \mathrm{~B}$ neutrinos off $\mathrm{Xe}$ isotopes. Results are given for $g_{\mathrm{A}}=1.0$ and $g_{\mathrm{A}}=0.7$.

\begin{tabular}{lcc}
\hline \hline Target & \multicolumn{2}{c}{$\langle\sigma\rangle\left(10^{-42} \mathrm{~cm}^{2}\right)$} \\
\cline { 2 - 3 } & $g_{\mathrm{A}}=1.0$ & $g_{\mathrm{A}}=0.7$ \\
\hline${ }^{128} \mathrm{Xe}$ & 1.22 & 0.70 \\
${ }^{129} \mathrm{Xe}$ & 21.1 & 12.2 \\
${ }^{130} \mathrm{Xe}$ & 2.15 & 1.26 \\
${ }^{131} \mathrm{Xe}$ & 30.9 & 18.2 \\
${ }^{132} \mathrm{Xe}$ & 3.63 & 2.15 \\
${ }^{134} \mathrm{Xe}$ & 5.97 & 3.60 \\
${ }^{136} \mathrm{Xe}$ & 12.1 & 6.83 \\
\hline \hline
\end{tabular}



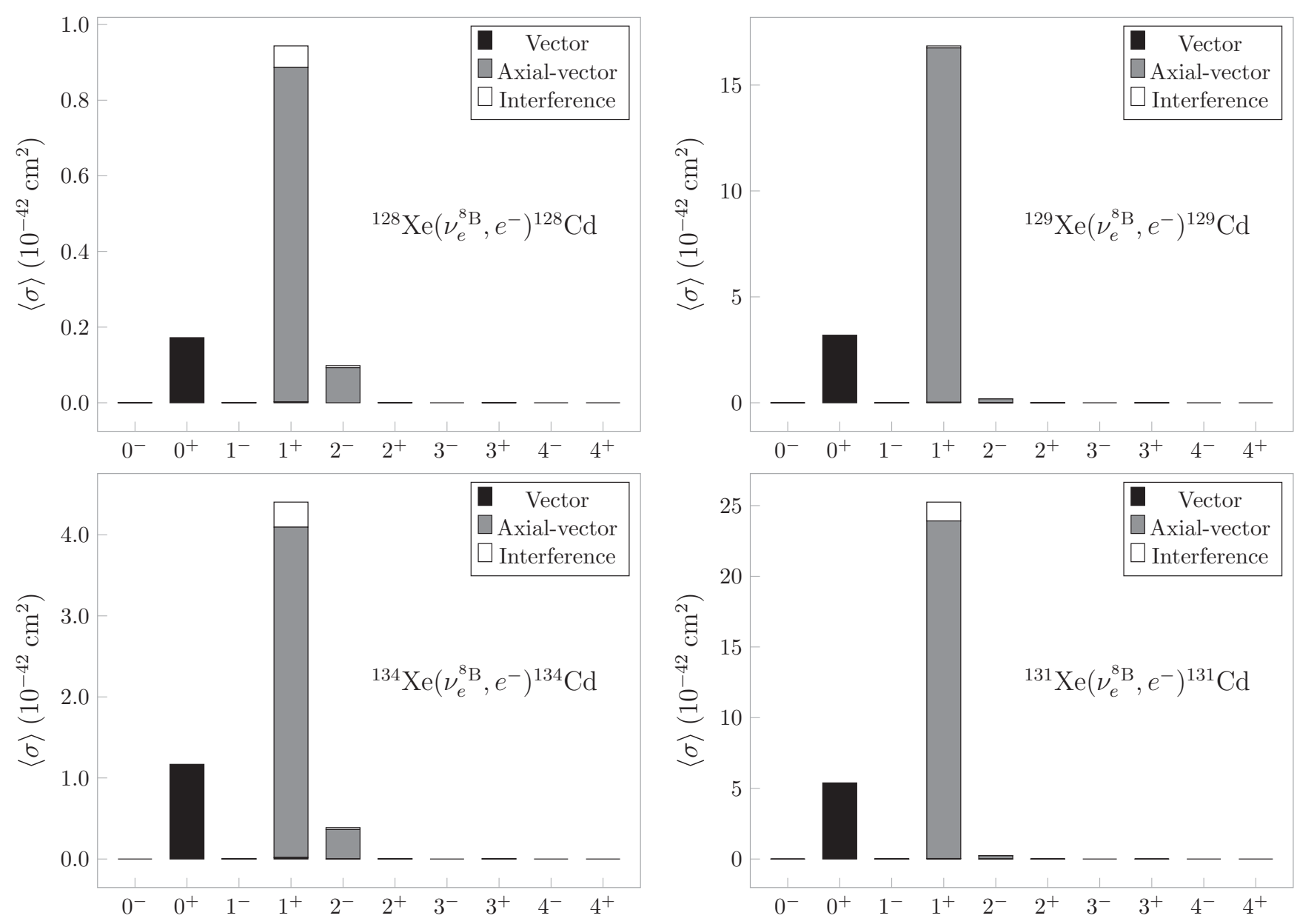

FIG. 5. Contributions of different multipole channels to the total folded cross section for ${ }^{8} \mathrm{~B}$ solar neutrinos scattering off a representative sample of Xe targets. Cross sections are given in units of $10^{-42} \mathrm{~cm}^{2}$ in all panels. Results are shown for ${ }^{128} \mathrm{Xe}$ (top left), ${ }^{129} \mathrm{Xe}\left(\mathrm{top}\right.$ right), ${ }^{134} \mathrm{Xe}$ (bottom left), and ${ }^{131} \mathrm{Xe}$ (bottom right). The calculations were made using $g_{\mathrm{A}}=1.0$.

\section{A. Supernova neutrinos}

Using the energy distributions described in Sec. II we have computed the total averaged cross sections for supernova (anti)neutrinos scattering off xenon targets. The total cross sections obtained for supernova neutrinos and antineutrinos are presented in Table IV. The computations were made using $g_{\mathrm{A}}=1.0$ and $g_{\mathrm{A}}=0.7$. The cross sections for supernova neutrinos have a rising trend as a function of the mass number within an isotopic chain of fixed $Z$ due to the behavior of the transition $Q$ value and the Ikeda sum rule as discussed earlier. For the odd-mass isotopes the cross sections are significantly larger than for their even-mass neighbors as could be expected for these average neutrino energies in the range of $10-20 \mathrm{MeV}$ by the analysis of Tables II and III earlier. In neutral-current processes similar behavior was noted in Ref. [33]. For antineutrinos the pattern is reversed and the cross section generally decreases with increasing $A$ as the behavior of the transition $Q$ value is now the opposite. Similar enhancement in the antineutrino-nucleus cross section is also noted for the odd-mass target nuclei.

In Fig. 1 we show the contributions to the total averaged cross section arising from different multipole channels for electron neutrinos scattering off a representative sample of ${ }^{128} \mathrm{Xe},{ }^{129} \mathrm{Xe},{ }^{131} \mathrm{Xe}$, and ${ }^{134} \mathrm{Xe} . g_{\mathrm{A}}=1.0$ was used in the calculations for this and the rest of the figures in this section. In all cases the most important contributions come from the $1^{+}$axial-vector channel. Notable contributions arise also from the $0^{+}$multipole via vector coupling, and the $2^{-}$axialvector channel. Figure 2 shows the same for antineutrinos. For antineutrinos the most important multipole is $1^{-}$for the even-mass isotopes, but $1^{+}$for the odd-mass isotopes. In the $1^{-}$multipole there are large contributions from both the vector and axial-vector channels. Also the interference between the vector and axial-vector parts gives a noticeable negative contribution to the antineutrino scattering cross section. The axial-vector contributions scale as $g_{\mathrm{A}}^{2}$, so quenching of the coupling constant could have a significant effect on the results in Fig. 1. Some change would also be induced to the small interference part, which has a linear dependence on $g_{\mathrm{A}}$.

It is interesting to see which final states contribute most to the total cross sections. We show the contributions arising from different final states of excitation energy $E_{\text {ex }}$ with respect to the $\mathrm{Cs}$ nucleus ground state in Fig. 3 in the reactions $\mathrm{Xe}+v_{e} \rightarrow \mathrm{Cs}+e^{-}$. Very large contributions come from 

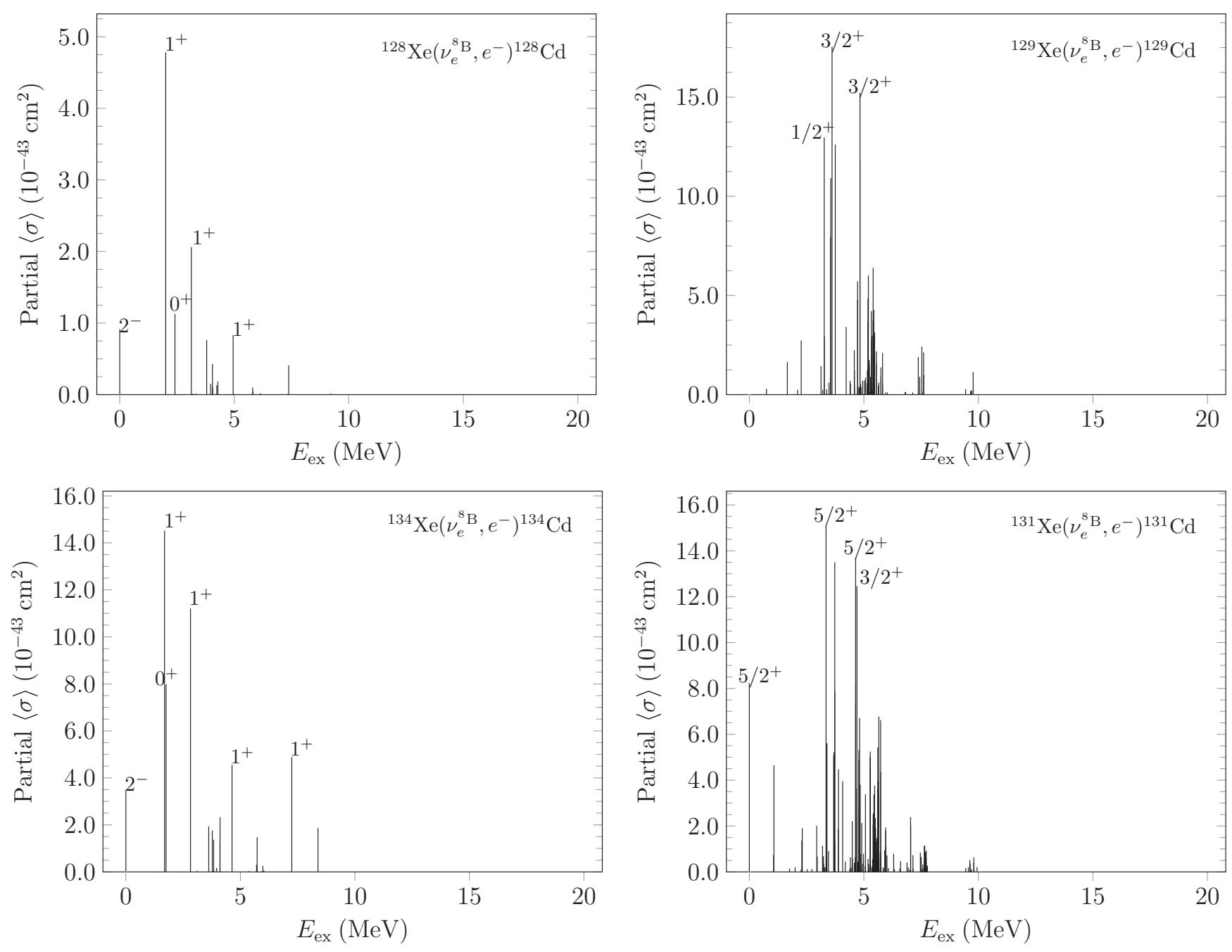

FIG. 6. Main contributions to the total averaged cross section arising from different final states of the reaction daughter Cs nuclei in the reactions ${ }^{A} \mathrm{Xe}+v_{e}^{8} \mathrm{~B} \rightarrow{ }^{A} \mathrm{Cs}+e^{-}$. Results are shown for $A=128$ (top left), $A=129$ (top right), $A=134$ (bottom left), and $A=131$ (bottom right). The calculations were made using $g_{\mathrm{A}}=1.0$.

the Gamow-Teller giant resonance state $1_{\mathrm{GTGR}}^{+}$and isobaric analog state $0_{\mathrm{IAS}}^{+}$for the even-mass xenon targets. In the case of ${ }^{128} \mathrm{Xe}$ the IAS is predicted to be quite low in energy by the empirical formula, which causes the contribution from the IAS to be more significant than that for ${ }^{134} \mathrm{Xe}$. Should the IAS be $2 \mathrm{MeV}$ higher for ${ }^{128} \mathrm{Xe}$ the effect in the total cross section would be roughly $5 \%$. For the odd-mass isotopes the main contributions arise from $1 / 2^{+}$and $3 / 2^{+}$states $(\Delta J=0,1$ with respect to the ground state) at roughly $5 \mathrm{MeV}$.

In Fig. 4 we show the total cross section to different final states for antineutrinos scattering off Xe nuclei in the reactions $\mathrm{Xe}+\bar{v}_{e}^{\mathrm{SN}} \rightarrow \mathrm{I}+e^{+}$. Here the most important contributions come from $1^{-}$states for the even-mass targets. The leading contribution for antineutrinos scattering off ${ }^{129} \mathrm{Xe}$ is from a $3 / 2^{-}$final state at roughly $7 \mathrm{MeV}$. Interestingly most of the cross section for the $A=131$ process is carried by the $5 / 2^{+}$ MQPM ground state of the resulting I nucleus. Experimentally, however, the ground state of ${ }^{131} \mathrm{I}$ is $7 / 2^{+}$, but there is a low-lying $5 / 2^{+}$state at roughly $150 \mathrm{keV}$.

\section{B. Solar ${ }^{8}$ B neutrinos}

We have also computed total averaged cross sections for electron neutrinos from ${ }^{8} \mathrm{~B}$ decay in the Sun. The ${ }^{8} \mathrm{~B}$ neutrino profile was taken from Ref. [42]. The spectrum of ${ }^{8} \mathrm{~B}$ neutrinos peaks at around $7 \mathrm{MeV}$, and thus the average energy is somewhat lower compared to the supernova neutrinos. The tail of the spectrum goes up to $16 \mathrm{MeV}$, so only few if any neutrinos can excite the important high-lying IAS and GTGR states.

The computed total averaged cross sections of the process ${ }^{A} \mathrm{Xe}+v_{e}^{{ }^{8} \mathrm{~B}} \rightarrow{ }^{A} \mathrm{Cs}+e^{-}$are given in Table V. Results are shown for $g_{\mathrm{A}}=1.0$ and $g_{\mathrm{A}}=0.7$. Due to the average energy of the neutrinos being lower than that of the supernova neutrinos, the cross sections are systematically an order of magnitude smaller for solar neutrinos. The contrast between even-mass and odd-mass isotopes is even more pronounced in the case of solar neutrinos. This is due to the fact that in the odd-mass nuclei final states that give large contributions to the cross sections are at easily accessible an energy even for solar 
neutrinos. Meanwhile for the even-mass nuclei some of the states that would have a strong transition are at the very end of the ${ }^{8} \mathrm{~B}$ energy spectrum leading to essentially zero probability of populating those states.

In Fig. 5 we show the contributions to the total averaged cross section arising from different multipole channels for the ${ }^{8} \mathrm{~B}$ solar neutrino case. We used $g_{\mathrm{A}}=1.0$ in the calculations for this and the following figures in this section. Figure 5 is very similar to Fig. 1 for supernova neutrinos, but here the $1^{-}$ and $2^{-}$contributions are smaller relative to the $1^{+}$contribution. Typically for low neutrino energies the $1^{+}$axial-vector channel dominates the cross section, but for high energies the neighboring multipoles also start to play a large role. The axial-vector contributions are of course smaller if substantial quenching of $g_{\mathrm{A}}$ is present.

It is interesting to compare the cross sections to different final states between solar neutrinos and the more energetic supernova neutrinos. The contributions from different final states for ${ }^{8} \mathrm{~B}$ neutrino scattering are shown in Fig. 6 for ${ }^{128} \mathrm{Xe},{ }^{129} \mathrm{Xe},{ }^{131} \mathrm{Xe}$, and ${ }^{134} \mathrm{Xe}$ targets. Here we notice that for the even-mass nuclei the major contributions come from states at much lower energies than for supernova neutrinos. In odd-mass nuclei the important bunch of states around $5 \mathrm{MeV}$ is well accessible for solar neutrinos, and many of the leading contributions arise from those states for both supernova and solar neutrinos. States above approximately $10 \mathrm{MeV}$ in the final nuclei have little to no contribution to the total cross section for solar neutrinos.

\section{CONCLUSIONS}

We have computed charged-current neutrino nucleus scattering cross sections for neutrinos scattering off ${ }^{128} \mathrm{Xe},{ }^{129} \mathrm{Xe}$, ${ }^{130} \mathrm{Xe},{ }^{131} \mathrm{Xe},{ }^{132} \mathrm{Xe},{ }^{134} \mathrm{Xe}$, and ${ }^{136} \mathrm{Xe}$. The wave functions of the involved nuclear states were obtained in the pnQRPA and MQPM frameworks. We have given the total cross section as a function of neutrino (antineutrino) energy in Table II (III), making it possible to obtain an estimate of total cross section for a desired neutrino energy distribution within the range of 5-80 MeV. Here we have used realistic energy profiles describing supernova (anti)neutrinos and neutrinos from ${ }^{8} \mathrm{~B}$ decay in the Sun.

The cross sections we obtained are considerably larger for scattering off the odd-mass nuclei ${ }^{129} \mathrm{Xe}$ and ${ }^{131} \mathrm{Xe}$ compared to the neighboring even-mass nuclei in the solar and supernova neutrino energy range. One contributing factor is the lower $Q$ value of the transition between odd-mass nuclei compared to the even-mass neighbors. Also the denser low-lying energy spectrum of odd-mass nuclei has an effect that increases the cross section for the average supernova neutrino energies. In Ref. [33] we noticed an anomalously large $0^{+}$multipole contribution in neutral current neutrinonucleus scattering off odd-mass Xe isotopes in the MQPM formalism, which might have explained in part the larger cross sections of odd-mass nuclei. Here, however, the cross sections are dominated by the $1^{+}$multipole as they should be and if spurious $0^{+}$contributions exist, they should be small.

The high-lying $0^{+}$isobaric analog state and especially the $1^{+}$Gamow-Teller giant resonance are very important to neutrinos scattering off even-mass nuclei. Solar neutrinos are too low in energy to cause excitation of these states in Xe nuclei. The odd-mass Xe nuclei, on the other hand, have many important final states at lower energies, which are accessible by solar neutrinos. Therefore, the cross sections are an order of magnitude larger for odd-mass targets rather than evenmass targets. Most of the solar neutrino scattering events observed in a natural xenon detector would thus be from interactions with ${ }^{129} \mathrm{Xe}$ and ${ }^{131} \mathrm{Xe}$.

Due to the difference in average neutrino energy, the computed total averaged cross sections are an order of magnitude smaller for solar ${ }^{8} \mathrm{~B}$ neutrinos than for supernova neutrinos. However, the solar neutrinos have a constant flux, which helps to build up events in a long-term experiment. Given that a supernova would happen close enough, the neutrino burst would be very intense but quite short in any case.

As a final note, we would like to return to the problem of neutrino mixing in the dense supernova environment. The effects caused by this mixing might be helpful in determining the neutrino mass hierarchy. A detailed study of neutrinomixing effects using recently obtained fluxes from state-ofthe-art supernova simulations would be an interesting continuation of the present study.

\section{ACKNOWLEDGMENTS}

P.P. was supported by a graduate student stipend from the Magnus Ehrnrooth Foundation. E.Y. thanks the financial support of the Grant No. 2016/25143-7 from the Sao Paulo Research Foundation (FAPESP).
[1] A. Dighe, J. Phys.: Conf. Ser. 136, 022041 (2008).

[2] P. D. Serpico, S. Chakraborty, T. Fischer, L. Hüdepohl, H. T. Janka, and A. Mirizzi, Phys. Rev. D 85, 085031 (2012).

[3] N. Ackerman et al. (EXO Collaboration), Phys. Rev. Lett. 107, 212501 (2011).

[4] M. Auger et al. (EXO Collaboration), Phys. Rev. Lett. 109, 032505 (2012).

[5] nEXO Collaboration, arXiv:1805.11142 [physics.ins-det].

[6] J. Monroe and P. Fisher, Phys. Rev. D 76, 033007 (2007).

[7] J. Billard, E. Figueroa-Feliciano, and L. Strigari, Phys. Rev. D 89, 023524 (2014).
[8] E. Aprile et al., Phys. Rev. Lett. 119, 181301 (2017).

[9] V. A. Kudryavtsev and for the LZ collaboration, AIP Conf. Proc. 1672, 060003 (2015).

[10] J. Aalbers et al., JCAP 11, 017 (2016).

[11] T. Suzuki, S. Chiba, T. Yoshida, T. Kajino, and T. Otsuka, Phys. Rev. C 74, 034307 (2006).

[12] C. Volpe, N. Auerbach, G. Colò, T. Suzuki, and N. Van Giai, Phys. Rev. C 62, 015501 (2000).

[13] W. C. Haxton, Phys. Rev. D 36, 2283 (1987).

[14] W. E. Ormand, P. M. Pizzochero, P. F. Bortignon, and R. A. Broglia, Phys. Lett. B 345, 343 (1995). 
[15] J. Kostensalo, J. Suhonen, and K. Zuber, Phys. Rev. C 97, 034309 (2018).

[16] T. Suzuki, M. Honma, K. Higashiyama, T. Yoshida, T. Kajino, T. Otsuka, H. Umeda, and K. Nomoto, Phys. Rev. C 79, 061603(R) (2009).

[17] E. Ydrefors and J. Suhonen, Adv. High Energy Phys. 2012, 373946 (2012).

[18] W. Almosly, E. Ydrefors, and J. Suhonen, J. Phys. G: Nucl. Part. Phys. 40, 095201 (2013).

[19] P. C. Divari, Adv. High Energy Phys. 2013, 143184 (2013).

[20] E. Ydrefors, J. Suhonen, and Y. M. Zhao, Phys. Rev. C 91, 014307 (2015).

[21] W. Almosly, E. Ydrefors, and J. Suhonen, J. Phys. G: Nucl. Part. Phys. 42, 095106 (2015).

[22] R. Lazauskas and C. Volpe, Nucl. Phys. A 792, 219 (2007).

[23] W. Almosly, B. G. Carlsson, J. Dobaczewski, J. Suhonen, J. Toivanen, P. Vesely, and E. Ydrefors, Phys. Rev. C 89, 024308 (2014).

[24] W. Almosly, B. G. Carlsson, J. Suhonen, J. Toivanen, and E. Ydrefors, Phys. Rev. C 94, 044614 (2016).

[25] M.-K. Cheoun, E. Ha, T. Hayakawa, T. Kajino, and S. Chiba, Phys. Rev. C 82, 035504 (2010).

[26] M.-K. Cheoun, E. Ha, K. S. Kim, and T. Kajino, J. Phys. G: Nucl. Part. Phys. 37, 055101 (2010).

[27] M.-K. Cheoun, E. Ha, T. Hayakawa, S. Chiba, K. Nakamura, T. Kajino, and G. J. Mathews, Phys. Rev. C 85, 065807 (2012).

[28] N. Paar, D. Vretenar, T. Marketin, and P. Ring, Phys. Rev. C 77, 024608 (2008).

[29] E. Kolbe, K. Langanke, and G. Martinez-Pinedo, Phys. Rev. C 60, 052801 (1999).

[30] E. Kolbe and K. Langanke, Phys. Rev. C 63, 025802 (2001).

[31] T. Suzuki and M. Honma, Phys. Rev. C 87, 014607 (2013).

[32] E. Ydrefors and J. Suhonen, Phys. Rev. C 87, 034314 (2013).

[33] P. Pirinen, J. Suhonen, and E. Ydrefors, Adv. High Energy Phys. 2018, 9163586 (2018).

[34] E. Kolbe, K. Langanke, G. Martínez-Pinedo, and P. Vogel, J. Phys. G: Nucl. Part. Phys. 29, 2569 (2003).
[35] J. Engel, Phys. Rev. C 57, 2004 (1998).

[36] J. D. Walecka, Theoretical Nuclear and Subnuclear Physics (Imperial College Press, London, 2004).

[37] J. S. O'Connell, T. W. Donnelly, and J. D. Walecka, Phys. Rev. C 6, 719 (1972).

[38] H. Duan and J. P. Kneller, J. Phys. G: Nucl. Part. Phys. 36, 113201 (2009).

[39] J. Gava and C. Volpe, Phys. Rev. D 78, 083007 (2008).

[40] A. B. Balantekin and G. M. Fuller, Phys. Lett. B 471, 195 (1999).

[41] J. N. Bahcall, A. M. Serenelli, and S. Basu, Astrophys. J. 621, L85 (2005).

[42] J. N. Bahcall, E. Lisi, D. E. Alburger, L. De Braeckeleer, S. J. Freedman, and J. Napolitano, Phys. Rev. C 54, 411 (1996).

[43] J. Suhonen, From Nucleons to Nucleus: Concepts of Microscopic Nuclear Theory (Springer, Berlin, 2007).

[44] A. Bohr and B. R. Mottelson, Nuclear Structure (Benjamin, New York, 1969), Vol. 1.

[45] Y. Xu, H. Guo, Y. Han, and Q. Shen, J. Phys. G: Nucl. Part. Phys. 41, 015101 (2014).

[46] K. Holinde, Phys. Rep. 68, 121 (1981).

[47] A. H. Wapstra and G. Audi, Nucl. Phys. A 432, 1 (1985).

[48] J. Suhonen, Frontiers Phys. 5, 55 (2017).

[49] P. Pirinen and J. Suhonen, Phys. Rev. C 91, 054309 (2015).

[50] F. F. Deppisch and J. Suhonen, Phys. Rev. C 94, 055501 (2016).

[51] P. Puppe, D. Frekers, T. Adachi, H. Akimune, N. Aoi, B. Bilgier, H. Ejiri, H. Fujita, Y. Fujita, M. Fujiwara, E. Ganioglu, M. N. Harakeh, K. Hatanaka, M. Holl, H. C. Kozer, J. Lee, A. Lennarz, H. Matsubara, K. Miki, S. E. A. Orrigo, T. Suzuki, A. Tamii, and J. H. Thies, Phys. Rev. C 84, 051305(R) (2011).

[52] J. Suhonen and O. Civitarese, Nucl. Phys. A 924, 1 (2014).

[53] O. Civitarese and J. Suhonen, Phys. Rev. C 89, 044319 (2014).

[54] Z. M. Niu, Y. F. Niu, H. Z. Liang, W. H. Long, and J. Meng, Phys. Rev. C 95, 044301 (2017).

[55] Y.-Z. Qian, W. C. Haxton, K. Langanke, and P. Vogel, Phys. Rev. C 55, 1532 (1997).

[56] J. Toivanen and J. Suhonen, Phys. Rev. C 57, 1237 (1998). 